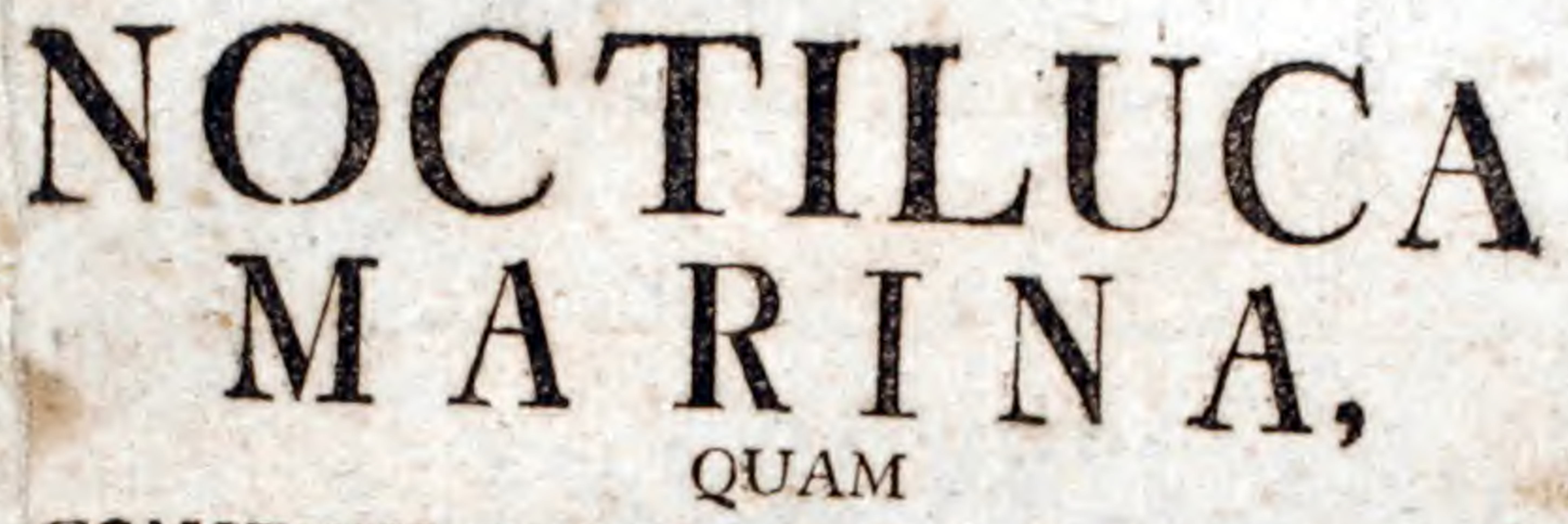

CONSENSU EXPERIENT. FACULT. MED: IN REG. ACAD. UPSALIENSI,

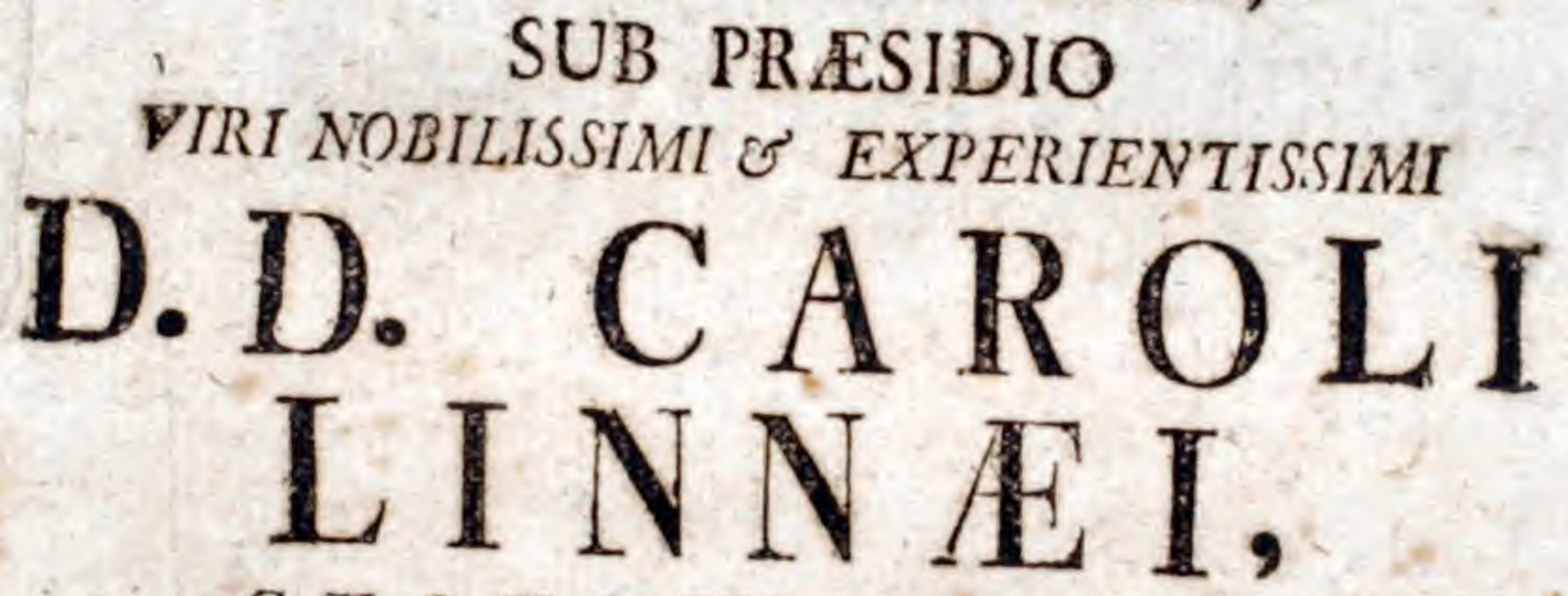

S:IE. R:IE. M:TIS. ARCHIATRI,

MED. ET BOTAN. PROFESS. REG ET ORD. Áad. Imper. N. C. Monspel. Berol. Tolos. STOCKH. ET UPSAL. SOCH,

CAROLUS FRID. ADLER IN AUD. CAROL. MAJ. D. I XJU A MDCCLII. HORIS ANRE MERIDIEN SOLITIS

UPSALI A

gXXGdir LAUR MAGN. HójER, REg. ACAD. TYP, 


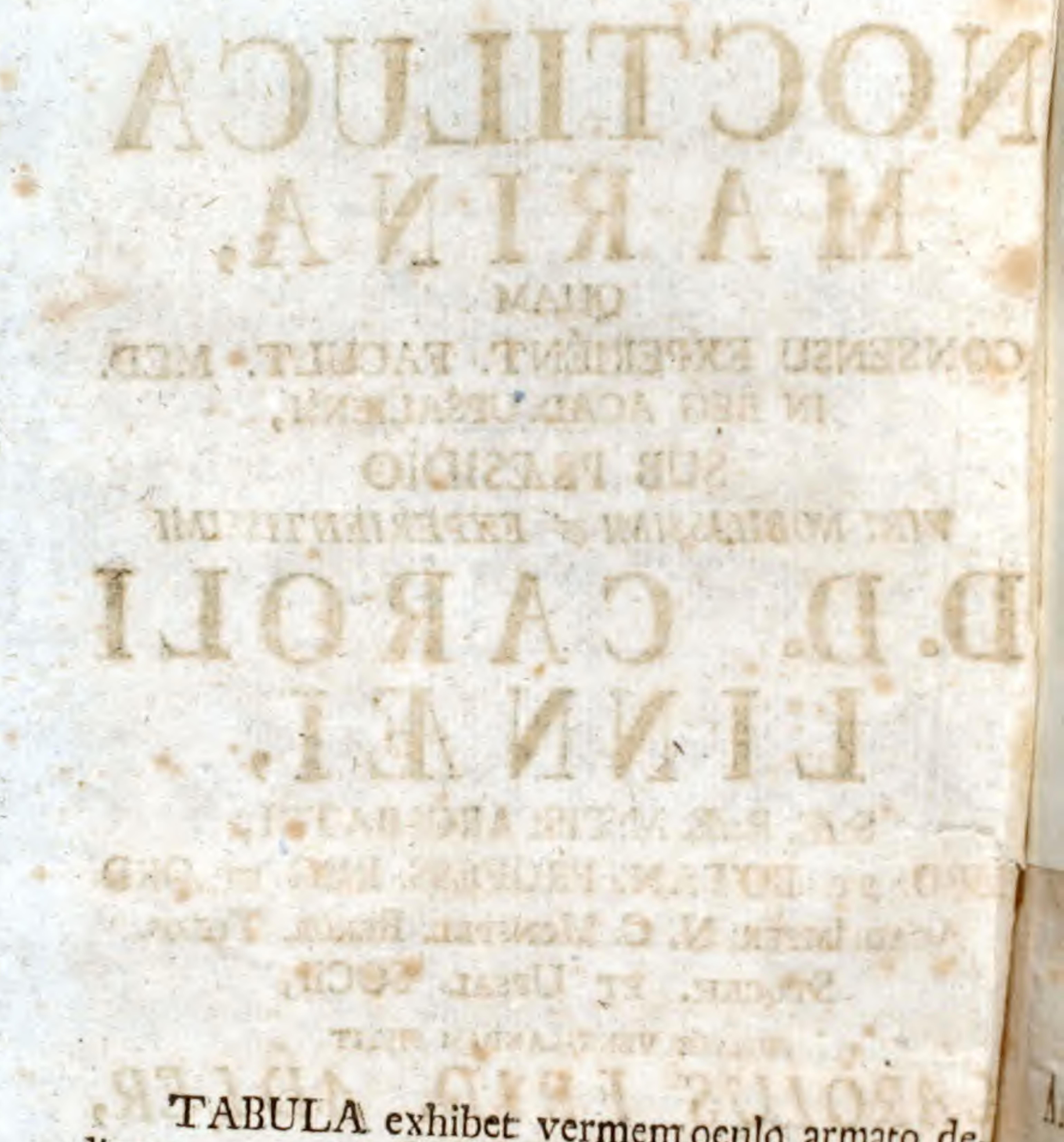

lineatum qui exhibet vermem oculo armato de. attingat. i vix lineam ,

\section{3. $1,8,920$}

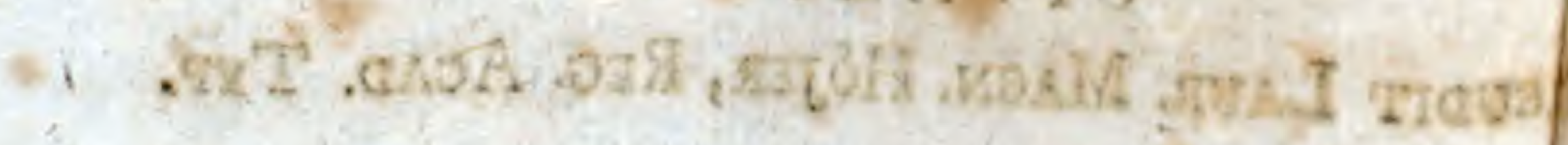




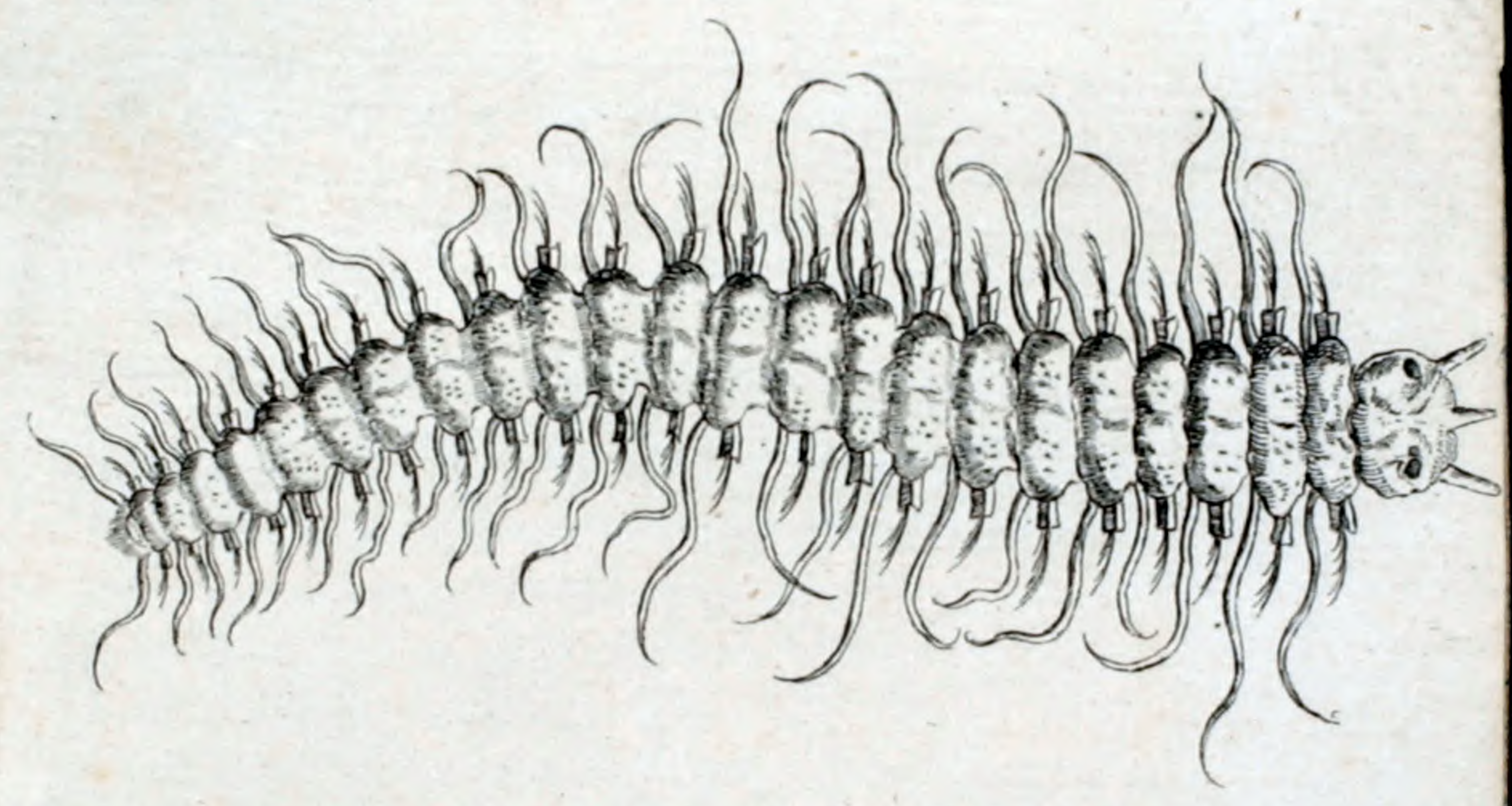





\section{à MON SIEUR \\ C OLIN CA M P E L L. CONSEILIER de Commerce, \\ DIRECTEUR de la compagnie des intes. \\ à MONSIEUR \\ WILHELM LAGERSTROM, \\ CONSETLLER de Commerce, \\ DIRECTEUR de la Compagnie des Indes. \\ à $\mathrm{MONSIEUR}$ \\ ABRAHAM GRIL L, MARCHAND, \\ DIRECIEUR de la Campagnie des Indes. \\ à MONSIEUR \\ NICOLAS SAHLGREN. \\ MARCHAND, \\ DIRECTEOR de la Compagnie des Iníe. \\ à MONSIEUR \\ ANDR. PLOMGREN, \\ MARCHAND, \\ DIRECTEUR de la Compagnie des Indes. \\ à MONSIEUR \\ C L A S GR I LL, \\ MARCHAND, proprietairede Mines, DIRECTEUR de la compagnie des Indes. MESSIEURS}




\section{MESSIEURS}

I E Monde fçavant vous cft redevable du plaifir qui il L a de fe voir enrictsi de quantité de Fxperiences interefantes, ES des belles collections dans $L$ bittoire Naturelle. C'eft pour le fervice de la Patrie que vous rifguez vos bicns; sar qui peut nicr, qu'un Commerce. nvantagenx ne foit un moyen jur de faire fleurir un Etar. Puis-ie avoir oublie, que c'eft presque á Vous fenls que ic fuis redevable de ma fortune, OS de la fatisfaction que ja a eu de contempler les merveilles de la nature dans les prïs tes plus reculés. Aggréez donc comme une marque de ma reconnoiflance cet aveu, qui laife entrevoir en ne.eine tếms combien les fciences ont tiré dutilité de la navigation. En Vous foubaitant toutes fortes de propperité, je regardersi conme mon bonbeur de poffeder Vos precieufes bien. veillances, E comme un banneur de pouvoir me dire

\section{MESSIEURS}

Votre tres humble \& tres obeiflant ferviteur OHARLES FRID. ADLER 

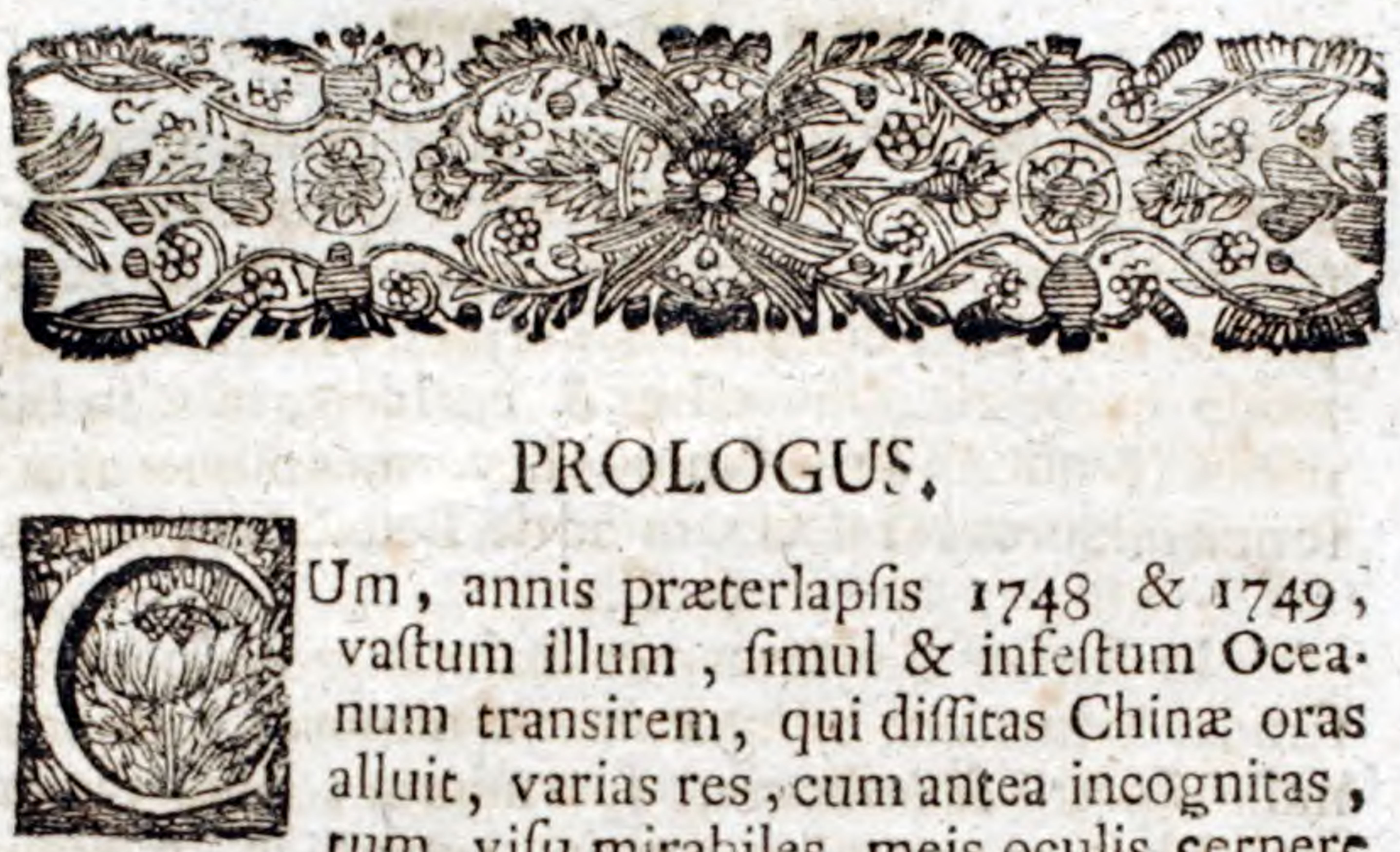

Um, annis præterlapfis $\mathbf{1} 748$ \& 1749 vaftum illum, fimul \& infertum Ocea. num transirem, qui diffitas Chinæ oras alluit, varias res, cum antea incognitas, tum vifu mirabiles, meis oculis cernere contigit, quarum omnium illud non pratereundum cenfeo, quod, nocturno tempore; aqua maritima, permodum fintillarum, non raro nituerit, ut fi in undis \& flammis igneis navigaremus.

Hoc monftrum fedulo quidem fum adniratus, tantum tamen abfuit, ut caufam hujusce indolis detegere valuerim, ut potius, opinioni nautarum indulgere coactus fuerim, qui ignem illum Typhoni. cos præfagire ventos fuperftitiofe conjecturabant.

Hre ergo res tantis involuta tenebris, eadem illa materies eft, cujus ego, primas jam lineas ducere, fpeciminisque loco, publico examini fubjicere mecum contticui; Sic navita de vencis.

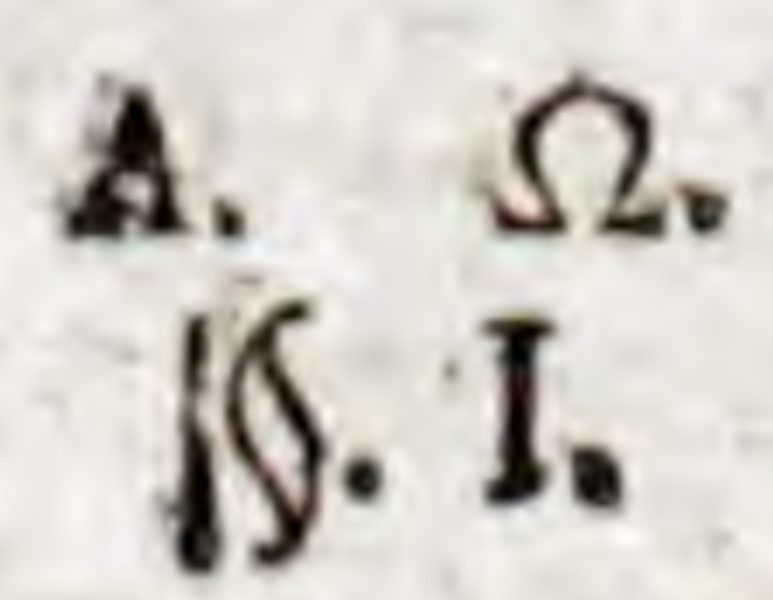

Mare, quod terre mater merito falutetur, "miII norem non poffidet Animalium mirabilium nuDLf merum, quam fuo portat gremio filia. Producic quippe maximas Balænas, voraciffimos Sqvalos, \& A in. 
innumerabilia reliqua pifcium mirabilium genera, quæ certius vidit, quam feliciter depinxit Valentyn. franc. in fua Ind. Orient. In ayua fua quafi acceperune don icilia communia Vermes, exceptis folummodo quibusdam limacibus \& cochleis, qux in humidis vivune umbris, funtque hi vermes numero for te non minores ac infecta, quod de Teftaceis vidimus. S. II.

In fenfus bumanos res majoris molis primum remper ineurrunt; reliquæ autem minores, tanquam nullius pretii \& utilitatis, fpernuntur; cum tamen rerum natura nunquam magis, quam in minimis tota fit. Hinc prifci revi homines res majores perpenderunt, minimas vero, ut nullius fere ufus, no. bis reliquere; Interea tamen laudatiffimi noftri tem. poris Botanici, qui intra feculum \& quod excurrit, doetrinam Bosanicam abfolverunt, etiam minimos mufcos jufta lance penficarune, qui in refpectu herbarum, utviliffini vermes ad animalia funt confiderandi. 5. III.

Vermium hiforia eft profecto ftupenda; Tremblaus, Renumur aliqune jam ante decennium, orbi demonftrarune primum admirabiles illos POLYPOS eorumque proprietates. D: ni Fuffieus \& Dohatus, intra ejusdem temnoris foatium, illos detegere cceperune vermes, qui COR AL LIA fabricant; fic qui jufta qua. dam curiofitate res ftupendas videre cupie, in maris inferiora defcendat, neceffe eft, \& inter vermes.

\section{S. IV.}

Ad fulgorem aque marinx gnod seinee, hujus rei folidam non hatuimas experientiam ante annum 


\section{(2)3) $) \circ($ (e)}

1750 , cum plenam occafionem naturæ peritis dederunt trali. Horum, in ferutandis naturz prodigiis, diligentia non minima fuic in prateritis temporibus, ut nec ultimum jam, de hiftoria vermium, locum merentur. Antiquiores illos, IMPERATUM loquor, COLUMNAM, ALDROVAN. DUM, BOCCONEM ficco, quod ajunt, ut prætereamus pede, nobis recordari fufficiat inter recentiores: BONANNI Recreationem mentis \& oculi. Rome 1681 MUSEUM Kirkerianum Roma 1709. fol. VALISNERII Opera Phyfico.Medica, venet. 1733: fol. vol. 3 .

PLANCI Librum de concbis minus notis. venet. 1739. GVALTIERI Indicem Conchyliorum. Florent. 1742. DONATI Hiftoriam Naturalem maris Adriacici

Vened. 1750.

Quorum omnium opera novis \& perfpicuis vermium obfervationibus impleta funt.

$$
\text { Q. V. }
$$

Quod fulgorem nitoremque fuum noctibus perfæpe, tam late ipargat aqua marina, quoad oculis illum cernere eft poffibile, præfertim undique eirca navem, inque xetuantibus \& fqualidis undis, res eft, non recentibus folum, verum antiquiffimis, notiffina.

\section{f. VI.}

VETERES, rerum non fatis periti, \& monftra è longinquo, h. e. ad corticem folummodo intuentes, illa fixe fabulis mirabilibus declarare tenta. runt. Sic ignem, jactata navi per turbines venti $\&$ aquæ, funibus \& aplauftris adfperfum, nec non 
ignem, noctibus tenebrofis, in mare ferventi lucentem \& non urentem, Caftorem nominarunt \& pollucem, quos in Deorum numerum pofuerunt, ut Plin, Lib. Il.Cap. XXVIII.

\section{VII.}

PHYCICI poftmodum inter Meteora illum afumferunt, usque ad Ds VIANELLI in fuo Nouwe fcoperte intorno le luci notturne dell aqua marina. Venet. 1749. p. 28 Tab. I. lumen hocce ex infinita minimorum vermium mulcitudine caufari demontravit, qui inermibus oculis vix cerni poffint.

\section{VIII.}

Hos novos \& mirabíles vermes nuper D:s GRISELINUS Obfervations fur la Scolopendre marine luilante. Vened. pag. 32 tab. 2. accuratius exploravit, defcripfit \& depinxit, auxilio Illuftrifimi, nec non, Scientiæ Naturæ maxime Eruditi,Equitis Gener.D. de . RATHGEL, Miniltre pour. L. L. M.M. Imperiales, refident aupres de la ferenifima Republ, de Venife, membr. de la Societ. Royale d'Upfal, cujus labore \& induftria factum, ut notitiam novi vermis, mare illuminantis, fibi acquifiverit orbis curiofus. Hic vermis f. Scolopendra marina lucens ejus. dem eft genéris, quod Nob. D. Prafes in fuo $S y / t$. Natur. edit. Holm. p. 72, fub nomine NEREISin lucem protulit.

\section{§. IX.}

Inter res quæ propriam lucendi vim habent; absque mutuatione ab aliis, numerantur: Sol \& Stellæ Ignis ordinarius, ignis ELECTRICUS, 


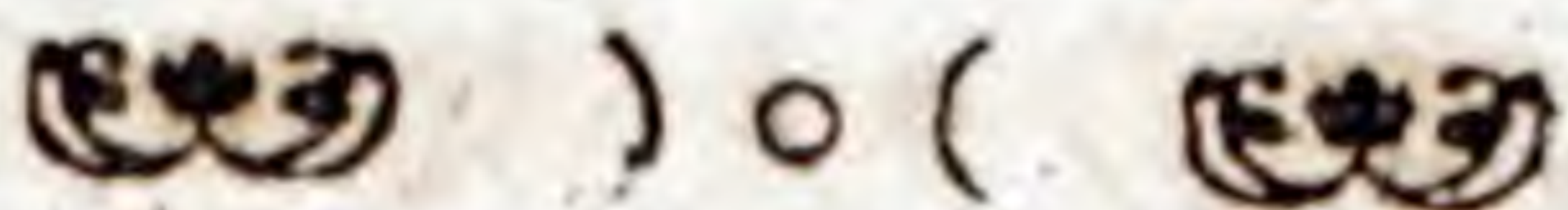

arte \& frictione productus, ignis facchari \& lapidis Calaminaris ex contritione,' pili variorum animalium ut Equorum \& Yelium cum dorfa manibus illinentur, PHOSPHORI artificiales, diverfo modo à Chymicis præparati, caro vitulina cum in cellaria, per aliquot dies, retinetur; pifces aliqui, ut Scomber, Gadus, Clupea, qui mortui aliquando in tenebris lue cent, ofla Efocis Acus dicti, BYSSUS VIOLACEA lignis adnafcens, fvetice ugle wed. Flor. fvec. I130, LAPIS Bononienfis \& 5 MURIA lapidea, Sy/t. Natur, 164, varia animalia viva, ut CICADA Laternaria. Faun. Svec. 645. ACt. Stockb. 1746. p. 60. SCOLOPENDRA, Faun. foec. 126r., CANTHARIS Faun. fvec. 584., oculi quarundam ferarum, ut Luporum, Buboe num aliarumque; præcipue vero lumen ejusmodi vermibus marinis ineft, ut SEPIÆ fpeciebus *), CONCHA valvis biantibus Chama vulgo ditfe, \& DA. CTYLI *) Balani etiam aliquid luminis habent noEtibus, in aëre frigido. FUCUS pennam referens, quem pifcatores Algierenfes interdum retibus extrahunt, fuum noctê lumen fpargit Cicindelæ inftar, ut proximiores pifces dignofci poffint.

Nereides Sertularia fabricantes, ut \& noftra fi non totum, at certe partem luminis fpargunt.
f. $\mathrm{X}$.

-) Plin, libr. IX. Cap LI. Dactylis eft natura in te. nebris, remoto fumine, alio fulgore clarere, \& quanto magis humorum habeant, ludere in ore madentium, lucere in manibus, atque etiam in folo \& vefte, decidentihus guttis.

*a A, ertis cultro anatomico Polypis, tantus effulcit fplendor, ut remota tempore vefpertino candela, Pala. 


\section{f. $\mathrm{X}$.}

Ad Chinam cum proficifcebatur- B. m. Tärnftrom noftras, qui anno 1746 proh dolor! ad Poli Candor d. 5. Decembris obdormuit, varia, noctí. bus in mare Indico, lumina lucentia videbat, qux Afterias five Medufas efle opinabatur, fed cum e. jusmodi venenatum ut putabant animal in navem demittere non paffi fint fuperftitiofi nautæ, in tene. bris illud relinquere cogebatur.

Idem illud animal in itinere meo anxie quidem quæfivi, fed res ad votum non evenit, aliis ergo exploratu relinquendum; utrum Jolephi Babaras hoc fit an non, alios inquirere concedam. Sxpe cum mari; profunditatem menfi fuerint nautæ, videbam particulas aliquot, fellarum inftar luminantium, minimæ magnitudinis, funiculo retracto affixas adhæ. rere, qux fimul ac in auras venere, ftatim pe. rierunt quæ quidem vermes proprii fui gene. ris procul dubio fuere.

\section{$\mathrm{f}$. XI.}

Sic lectorem meum ad contemplationem ani. malis cujusdam de noviffimis Orbi cognitis, quod in tenebris lucet, excitare mihi contigic; eam tamen controverfiam non meam facio: utrum omnis

aqua

tium Pifanum tum temporis affignatum, ardere sideretur ut intranti fubito timorem incuteret. Lumen ma. ximum quoddam fub cute latens, non caro ipfa emifit, \& quo vicinior putedrini pifcis, eo luxit ardentius. Unguibus digitisque picci admotis, ita fplentor adhafit, ut quali totiden ftellis finguli fulgerent. Barthol. 


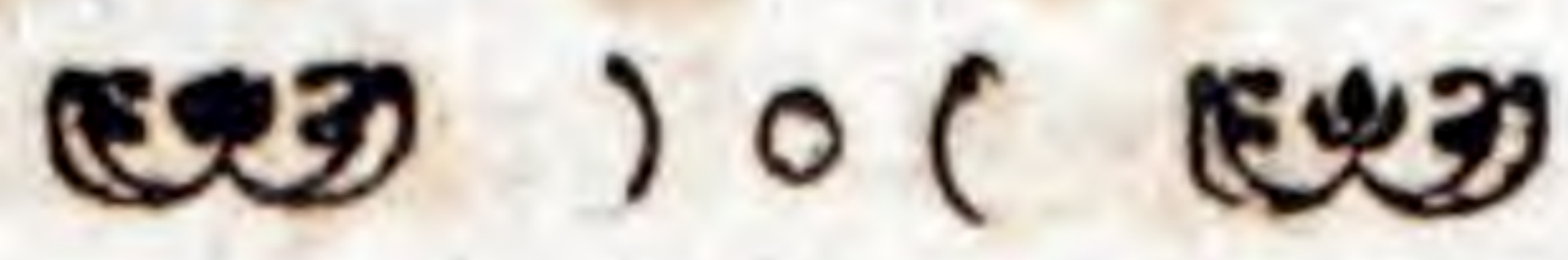

aqua maris illo luceat, an proprio fuo Sal gaudeat lumine; etenim me non fugit Dm. le Roy Parifinum demonftrare tentaffe aquam marinam de fe ipfo lucere, idque fequentibus argumentis: æatuantes maris fluctus lucidi, ampla illius fuperficies noctu" fepe fulgens, veftigia navium corufea, ipfam na." vem circumfluentes undæ, radiofa remigia, quo." rum pars immerfa folendet emerfa, aqua marina" cum in tenebris effunditur, quafi lumine plena, linteum gutris ejus irrotatum, poftea manibus fri-“6 catum, per fpatia irradians, cijus rei nuperrime per literas ad Nobiliffimum Dn. Prafidem mentio. nem fecit Gallix Decus.

\section{†. XII.}

Omittamus iftud Problema, an aliis quam vermibus luceat aqua marina, interea omnem no. bis fuftulerune dubitationem oculatiffimi Itali, quod de vermibus illam lucere poffibile fit; \& ut mini$\mathrm{mi}$ fint in mundo, ita cos innumerabiles credimus, quia quo minor eft fpecies creaturarum, eo major ejus mulciplicatio.

\section{§. XIII. \\ Cum jam Italorum libri in Hiftoria Naturali ad} noftras hand femper perveniant regiones, dulce mihi erit officium, animal tam novum tamque multi. plex eariofis naturæ cultoribus iterum proponere: quo noftrates excit nter ad examen hujus phonomeni, ide oque etiam D. Grifellini defcriptionem \& figus ram hic adjiciam: 
NEREIS phofphorans

Luccioletta dell' aqua marina Vianilli.

Scolopendre marine Luilante Grifelliwi.

CAPUT fubrotundum, depreffum toftro acuminato: TENTACULA duo, breviora, fubulat3.

OCULI duo, prominentes, ad latera capitis.

CORPUS conftans fegmentis viginti tribus, verfus pofteriora fenfim minoribus; fingula Segmenta ad lakus inftructa A pice conico, truncato, denticulato, e quo prodit Fafciculus pilorum, fub fingulo faificulo pilorum producitur filamentum pedes mentiens, fubulas tum, flexile, inerme.

COLOR animalculi hyalinus, pellucidus.

Magnitudo vix duas attingit lineas.

Habitat in plantis marinis, ut in Fucis or Algis, fepe ete-

jam plantas relinquit, $\circlearrowleft$ per maris /uperficiem vagatur 6. XIV.

Omni anni tempore animalculum hoc frequens eft, prefertim vero tempore æftivo \& inminente tempeftate, tunc enim ob inquietudinem veluti fuam majus lumen fpargit. Ventis ab auftrali Euro arl Eurum fpirantibus præcipue lucet. Majorem etiam lucendi vim habere videtur noctibus hiemalibus, cum dies calidus preivit. Si aqua de his vermibus lucens, vafi quodam inclufa, tepila teneatur usque ad putrefcentiam, duobus totis diebus lucet; aêre vero frigido non ultra octo horas. Sefe movendo lucent hi vermes, \& cum ex calore plus vigoris \& vivacitatis nancifcuntur, eo tuncmagis lucent. Quamprimum tangitur aqua, cui in funt hi vermes, mobilitate illorum mox lucet, ut \& fulcus, quem mari navigando infindit navis, ignis inftar fulgurat. Pifcatores etiam hoc luinen cernunt, retractis aëre calido, retibus $\mathrm{ex} \mathrm{aqua,} \mathrm{in} \mathrm{aquis} \mathrm{vero} \mathrm{bo} \mathrm{realibus} \mathrm{minus} \mathrm{frequens} \mathrm{hoc} \mathrm{phenomen} \mathrm{on}$ Ut \& in oceano reptentrionali hunc fulgoten parcius vidit $\mathrm{el}$. Prof. K $\$$ mius, qrume conträrio oceanus Africanus inter infulas Hefperidum \& $\mathrm{C}_{3}$. put bonæe fpei,fimul ac mare Indicum uberrima hobis offeruntejus raodis lumina, quod fiperflue me docuit autopfia.

I A N T U M. 